\title{
Optimizing Mission Critical Data Dissemination in Massive IoT Networks
}

\author{
Muhammad Junaid Farooq*, Hesham ElSawy ${ }^{\dagger}$, Quanyan Zhu*, and Mohamed-Slim Alouini ${ }^{\dagger}$, \\ *Department of Electrical \& Computer Engineering, Tandon School of Engineering, \\ New York University, Brooklyn, NY, USA, \\ ${ }^{\dagger}$ King Abdullah University of Science and Technology (KAUST), Thuwal, Saudi Arabia, \\ Emails: \{mjf514,qz494\}@nyu.edu, \{hesham.elsawy, slim.alouini\}@kaust.edu.sa.
}

\begin{abstract}
Mission critical data dissemination in massive Internet of things (IoT) networks imposes constraints on the message transfer delay between devices. Due to low power and communication range of IoT devices, data is foreseen to be relayed over multiple device-to-device (D2D) links before reaching the destination. The coexistence of a massive number of IoT devices poses a challenge in maximizing the successful transmission capacity of the overall network alongside reducing the multihop transmission delay in order to support mission critical applications. There is a delicate interplay between the carrier sensing threshold of the contention based medium access protocol and the choice of packet forwarding strategy selected at each hop by the devices. The fundamental problem in optimizing the performance of such networks is to balance the tradeoff between conflicting performance objectives such as the spatial frequency reuse, transmission quality, and packet progress towards the destination. In this paper, we use a stochastic geometry approach to quantify the performance of multi-hop massive IoT networks in terms of the spatial frequency reuse and the transmission quality under different packet forwarding schemes. We also develop a comprehensive performance metric that can be used to optimize the system to achieve the best performance. The results can be used to select the best forwarding scheme and tune the carrier sensing threshold to optimize the performance of the network according to the delay constraints and transmission quality requirements.
\end{abstract}

\section{INTRODUCTION}

The Internet of things (IoT) is enabling an ever increasing number of services and applications that is revolutionizing the way we interact with our surroundings [1]. The ability to remotely monitor and manage objects in the physical world is leading to a paradigm shift in sectors such as transportation, healthcare, public safety, energy management, home and industrial automation, etc. [2]. The future of IoT lies in ubiquitous sensing and connected systems that will provide enhanced situational awareness, data driven decision analytics, and automated response without human intervention [3]. The number of IoT devices 1 is growing at an increasing pace leading towards what is being referred to as the massive IoT [4]. It is estimated that the number of IoT devices will reach 1.5 billion in 2022 [5]. There is a wide variety of data carried over IoT networks, some of which might be mission critical such as in applications that require real-time monitoring and control. Hence, the successful transport of data

\footnotetext{
${ }^{1}$ We refer to both sensors and actuators as IoT devices.
}

with minimum delay from the source to destination is highly essential for effective operation of massive IoT systems.

Despite the fact that most IoT networks are infrastructure based, i.e., the sensors and actuators communicate with and are controlled by access points (APs) or base stations, the connectivity in these networks relies heavily on the use of device-to-device (D2D) [6] or machine-to-machine (M2M) [7] communications. This is mainly due to the following two reasons: (i) The low power remotely deployed IoT devices have limited communication range; therefore, the sensor data and control commands may have to traverse several D2D links to reach one of the APs. (ii) In certain application scenarios such as in geographically remote areas or battlefields [8], the traditional communication infrastructure such as cellular networks might be unavailable, which necessitates the use of D2D communication. Furthermore, simultaneous transmission by the massive number of devices requires medium access control (MAC) to regulate limited spectrum access. Contention based medium access protocols such as the carrier sense multiple access (CSMA) used for WiFi transmissions is well known to improve performance alongside providing equal opportunity of transmission to each device in the long run. Although efficient MAC design for IoT is still an active area of research, the contention based schemes are the most practical and viable ones [9]. In fact, many existing IoT devices either use traditional WiFi or are designed based on the IEEE 802.11 MAC protocol.

Contention based MAC protocols coordinate spectrum access among neighbouring devices to allow only nonoverlapping spatial transmissions to alleviate dominant interferers. The number of simultaneous transmitters in the network is controlled by a critical design parameter referred to as the carrier sensing threshold (CST) or detection threshold [10]. Increasing the CST enables more aggressive reuse of the spectrum over the spatial domain as more devices can transmit simultaneously. However, it also increases the interference which degrades the chances of transmission success. Hence, the CST imposes a delicate tradeoff between the transmission quality, measured by the probability of transmission success, and the spatial frequency reuse (SFR), quantified by the spatial density of concurrent transmitters [11]. This performance tradeoff has been well recognized in literature and attempts have been made to maximize the spatial density of successful 
transmissions by tuning the CST [12].

The efficient data dissemination in the case of mission critical massive IoT networks imposes additional performance objectives beyond maximizing the density of successful transmissions. In mission critical applications, the message transfer delay is crucial which requires the introduction of packet progress into the performance metric [13]. Although a metric known as the density of progress, which measures the mean number of meters progressed by all transmissions per unit area, has been introduced for the case of ALOHA medium access [12], it does not guarantee multi-hop progress from the source towards a particular destination. Moreover, the inherent intractability in analyzing the performance of CSMA inhibits further intricate analysis and hence, the notion of packet progress has not been investigated in this case. For CSMA networks, most analytical results in literature are presented for fixed transmitter-receiver distance in a single hop setting [14][16]. The model in [17], [18] studies multi-hop CSMA for vehicular networks; however, the developed models are only suited for one dimensional scenarios.

Transmitting data over multiple hops from source to destination requires selection of relay devices at each hop. In general, there can be numerous strategic ways to select potential forwarding devices among the neighbours; however, we investigate the extreme cases as used in [19], whereby the devices select the relay which results in the most progress towards the destination or the device which is the nearest to the transmitter. It helps in establishing meaningful performance bounds for the system. These forwarding strategies are referred to as the Most Forward with Fixed Radius (MFR) and Nearest with Forward Progress (NFP) respectively. In addition, we also study the intermediate benchmark case where a relay is selected at random from one of the available forward neighbours. This strategy is referred to as Random with Forward Progress $(R F P)$. The MFR strategy results in minimum number of hops to reach the destination, however, it may require multiple retransmissions due to low success probability that emanates from the distance dependent power decay. On the contrary, NFP strategy may require more number of hops to reach the destination but less retransmissions due to the high success probability resulting from shorter hop distance. The RFP may have a mix of long and short hops resulting in a mid point performance between the MFR and NFP. It is important to note that there is an interplay between the packet forwarding scheme and the CST. For instance, to maintain the same success probability, a forwarding scheme that relies on longer hops requires more conservative CST than the scheme that relies on shorter hops. Therefore, it is imperative to develop a comprehensive performance metric that can accurately capture these tradeoffs to optimize mission critical data dissemination in massive IoT networks.

In this paper, we exploit tools from stochastic geometry to model the system performance under the aforementioned packet forwarding schemes, namely the MFR, NFP, and RFP. The main performance parameters are the transmission success probability, the density of concurrent transmitters, and the

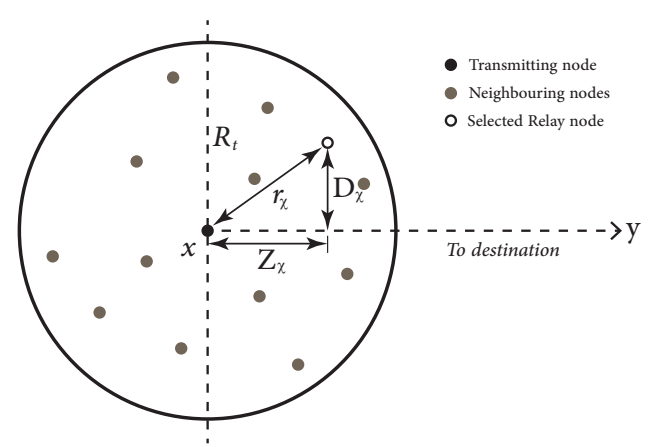

Fig. 1: Packet forwarding at each hop. The transmitter node at location $x$ forwards the packet to one of its forward neighbours based on the transmission scheme. The forward progress is the distance covered by the packet towards the destination node at location $y$.

forward packet progress. To simultaneously capture the effect of these three inter-related parameters, we use the average packet progress (APP) metric defined as the average successful progress made by packets towards the destination per unit area. The results show that the choice of forwarding scheme and the carrier sensing threshold that maximizes the average packet progress depends on the delay tolerance and the transmission quality requirements.

\section{SySTEM MOdEL}

We consider a network of uniformly scattered IoT devices in $\mathbb{R}^{2}$ represented by a marked homogeneous Poisson Point Process (PPP) $\Phi=\left\{X_{i}, M_{i}, T_{i}\right\}_{i \geq 1}$ of intensity $\lambda$, where the tuple $\left\{X_{i}, M_{i}, T_{i}\right\}$ denotes, respectively, the location, i.e., spatial coordinates, mark, and status of the $i^{\text {th }}$ device. The marks $M_{i} \in[0,1]$ are assumed to be independent and identically distributed (i.i.d.) uniform random variables, referring to the backoff timer value in the contention-based CSMA MAC protocol. The status $T_{i}$ is a Boolean value, which is 1 if the device at $X_{i}$ transmits and is 0 otherwise. According to the contention process, $T_{i}$ will be 1 only if the $i^{\text {th }}$ node has the least mark in its average contention range denoted by $\bar{R}_{s}$, i.e., $M_{i}=\min _{j}\left\{M_{j}:\left\|X_{i}-X_{j}\right\| \leq \bar{R}_{s}, j \geq 1\right\}$, where $\|\cdot\|$ is the Euclidean norm.

We assume that all devices transmit with a fixed transmit power $P$ and the power decays with the distance $r$ according to the power law $r^{-\eta}$, where $\eta>2$ is the path loss exponent. The power received from a transmitting device at $X_{i}$ by the receiver at $X_{j}$ is therefore evaluated as $P h_{i j}\left\|X_{i}-X_{j}\right\|^{-\eta}, i \neq j$, where $h_{i j}$ is the random channel fading gain between locations $X_{i}$ and $X_{j}$. We assume that the channel gains $h_{i j}$ are i.i.d. exponential random variables with mean $\mu^{-1}$. According to the block fading channel model, the channel gains are assumed fixed for the duration of each time slot and independent of the other time slots. The transmission range of the transmitter denoted by $R_{t}$ is defined as $R_{t}=\left\{\left\|X_{i}-X_{j}\right\|: P h_{i j} \| X_{i}-\right.$ $\left.X_{j} \|^{-\eta} \geq \rho_{\text {min }}, \forall i, j \geq 1\right\}$, where $\rho_{\min }$ is the minimum signal power for detection. The average transmission range can be expressed as $\bar{R}_{t}=\left(\frac{P}{\mu \rho_{\min }}\right)^{\frac{1}{\eta}} \Gamma\left(1+\frac{1}{\eta}\right)$. Similarly, the contention range $R_{s}$ is defined as $R_{s}=\left\{\left\|X_{i}-X_{j}\right\|: P h_{i j} \| X_{i}-\right.$ $\left.X_{j} \|^{-\eta} \geq \rho_{t h}, \forall i, j \geq 1\right\}$, where $\rho_{t h}$ is the CST, which is a 
central design parameter in this paper. The average contention range can be expressed as $\bar{R}_{s}=\left(\frac{P}{\mu \rho_{t h}}\right)^{\frac{1}{\eta}} \Gamma\left(1+\frac{1}{\eta}\right)$.

Fig. 11 illustrates an example realization of the network with uniformly deployed devices in $\mathbb{R}^{2}$. Note that only the selected transmitting device located at $x$ and its neighbors inside the average transmission range are shown. The data originates from the device placed at $x$ to a device or access point located at $y$ arbitrarily far from the transmitter. The transmitter at $x$ selects one of its available neighbors as a relay and forwards the data. The choice of next hop relay is determined by the forwarding scheme and is restricted to the forward direction only, i.e., towards the destination. The perhop transmitter-receiver separation, denoted by $r_{\chi}$, is a random variable and depends on the adopted forwarding scheme $\chi$, where $\chi \in\{M, N, R\}$ referring to the $M F R, N F P$ and $R F P$ strategies respectively. At each hop, a similar situation is encountered where the relay becomes the transmitter and hence, it is sufficient to analyze single hop performance.

We assume saturation conditions in which all devices always have backlogged data in their buffers, and hence, all of them will contend for spectrum access in each time slot ${ }^{2}$ At a given time slot, only the nodes that are qualified under the contention based MAC, i.e., $\left\{X_{i} \in \Phi: T_{i}=1\right\}$. The value of $T_{i}$ is determined by the contention domain of $X_{i}$, i.e., $\mathcal{N}_{i}=\left\{X_{j}: P h_{i j}\left\|X_{j}-X_{i}\right\|^{-\eta} \geq \rho_{t h}, \forall j \geq 1, j \neq i\right\}$. It is clear that $\rho_{t h}$ controls the cardinality of the contention domain denoted by $\mathbb{E}\left|\mathcal{N}_{i}\right|$ for all $X_{i} \in \Phi$. The set of concurrent transmitting nodes selected by the CSMA protocol is denoted by $\tilde{\Phi}=\left\{X_{i} \in \Phi: T_{i}=1\right\}$, and is modeled using a Matérn hard core point process (MHCPP). The MHCPP is obtained by a dependent thinning of the PPP $\Phi$ to obtain $\tilde{\Phi} \subseteq \Phi$. The resulting non-homogeneous PPP can be reasonably approximated by a homogeneous PPP with the following intensity [21]:

$$
\Lambda\left(\rho_{t h}\right)=\lambda \frac{1-e^{-\mathbb{E}\left|\mathcal{N}_{i}\right|}}{\mathbb{E}\left|\mathcal{N}_{i}\right|},
$$

where $\mathbb{E}\left|\mathcal{N}_{i}\right|$ is the expected number of nodes in the contention domain of $X_{i}$. In our case, it is equivalent to the device density times the area of the contention domain i.e. $\mathbb{E}\left|\mathcal{N}_{i}\right|=\lambda \pi \bar{R}_{s}^{2}, \forall i \geq 1$. Note that $\mathbb{E}\left|\mathcal{N}_{i}\right|$ is a function of $\bar{R}_{s}$ which in turn is a function of the $\operatorname{CST} \rho_{t h}$.

\section{ANALYSIS}

In this section, we first characterise the transmission quality at each hop in terms of the probability of transmission success for a fixed transmitter-receiver distance. We then provide the distribution of distances in the three packet forwarding strategies. Finally, we evaluate the distributions for the forward packet progress achieved by using each of the forwarding strategies.

\section{A. SINR Characterization}

The successful data delivery between the transmitter and receiver is dependent on the signal-to-noise-plus-interference-

\footnotetext{
${ }^{2}$ Extension to unsaturated buffers is straightforward and is dealt in |20|.
}

ratio (SINR). We use a signal capture model at the receiver with a detection threshold $\beta$, i.e., a transmission is successful or correctly decoded by the receiver only if the SINR is above the detection threshold $\beta$, which signifies the quality of service (QoS) requirement of the network. Without loss of generality, we can consider the receiving node to lie on $(0,0)$ in $\mathbb{R}^{2}$. Since the per-hop transmitter-receiver separation distance depends on the forwarding strategy $\chi$, each one of the strategies has a different SINR that is described as follows:

$$
\operatorname{SINR}_{\chi}=\frac{P h_{i 0} r_{\chi}^{-\eta}}{\kappa+I_{\tilde{\Phi} \backslash X_{i}}},
$$

where $\kappa$ is the thermal noise power and $I_{\tilde{\Phi} \backslash X_{i}}$ is the aggregate interference power emanating from the set of simultaneous transmitters excluding the tagged transmitter $X_{i}$, defined as $I_{\tilde{\Phi} \backslash X_{i}}=\sum_{j=\left\{k \geq 1: X_{k} \in \tilde{\Phi} \backslash X_{i}\right\}} P h_{j 0}\left\|X_{j}-0\right\|^{-\eta}$. The probability of transmission success for the forwarding strategy $\chi$ can be expressed as follows:

$$
\begin{aligned}
\mathcal{P}_{\chi} & =\mathbb{P}[S I N R>\beta]=\int_{r} \mathbb{P}\left[\frac{P h_{i 0} r^{-\eta}}{\kappa+I_{\tilde{\Phi} \backslash X_{i}}}>\beta\right] f_{r_{\chi}}(r) d r, \\
& =\int_{r} \exp \left\{-\frac{\mu \beta \kappa r^{\eta}}{P}\right\} \mathcal{L}_{I_{\tilde{\Phi} \backslash X_{i}}}\left(\frac{\mu \beta r^{\eta}}{P}\right) f_{r_{\chi}}(r) d r
\end{aligned}
$$

where $\chi \in\{M, N, R\} . \mathcal{L}($.$) is the Laplace transform (LT)$ of the probability density function $(p d f)$ of $I_{\tilde{\Phi} \backslash X_{i}}$. From (3), it is clear that we have to obtain the LT of the aggregate interference as well as the $p d f$ of $r_{\chi}$ in order to derive the probability of successful transmission. For tractability of analysis, the interference seen from the MHCPP $\tilde{\Phi}$ can be approximated by the interference seen from an equi-dense PPP [15]. Therefore, the LT of of aggregate interference is derived as follows:

$$
\begin{aligned}
& \mathcal{L}_{I_{\tilde{\Phi} \backslash X_{i}}}(s)=\mathbb{E}\left[e^{-s I_{\tilde{\Phi} \backslash X_{i}}}\right]=\mathbb{E}\left[e^{-s \sum_{j=\left\{k: X_{k} \in \tilde{\Phi} \backslash X_{i}\right\}} P h_{j 0} v^{-\eta}}\right], \\
& =\exp \left(-2 \pi \Lambda \int_{\phi \in \mathbb{R}^{2}}\left(1-\mathcal{L}_{h_{j 0}}\left(s P v^{-\eta}\right)\right) v d v\right) \text {, } \\
& \geq \exp \left(-2 \pi \Lambda \int_{\bar{R}_{s}-r}^{\infty} \frac{1}{1+\frac{\mu}{P s v^{-\eta}}} v d v\right),
\end{aligned}
$$

Note that integrating over the exact interference region denoted by $\phi$ in (4) is not possible in closed form due to its geometry with respect to the origin. However, observing that the nearest interferer can be located at a distance of $\bar{R}_{s}-r$ from the origin, a lower bound can be obtained by using the overestimated interference region as $\phi=\mathbb{R}^{2} \backslash b\left(0, \bar{R}_{s}-r_{\chi}\right)$, where $b(x, y)$ is a ball of radius $y$ centered at $x$. The lower bound of the LT in (5) can be expressed in closed form for $\eta=4$. In this case, the probability of transmission success can be obtained by numerically computing the following integral:

$$
\mathcal{P}_{\chi} \stackrel{(\eta=4)}{=} \int_{0}^{\bar{R}_{t}} e^{-\frac{\mu \beta \kappa r^{4}}{P}} e^{-\pi \Lambda \sqrt{\beta} r^{2} \tan ^{-1}\left(\frac{\sqrt{\beta} r^{2}}{\left(\bar{R}_{s}-r\right)^{2}}\right)} f_{r_{\chi}}(r) d r
$$


In the following subsection, we provide the $p d f \mathrm{~s}$ of the transmitter-receiver distance at each hop for the three main packet forwarding strategies, which is required to compute the probability of successful transmission.

\section{B. Distance Distribution}

The distance between transmitter and receiver at each hop depends on the choice of selected relaying devices at each hop. For the MFR forwarding scheme, the transmitter-receiver distance is denoted by $r_{M}$ and its distribution can be expressed by the following lemma:

Lemma 1: The distance between the transmitter and selected relay that maximizes the forward progress within the average transmission range $\bar{R}_{t}$ can be expressed as follows:

$$
\begin{aligned}
& f_{r_{M}}(r)=\int_{-\frac{\pi}{2}}^{\frac{\pi}{2}} \frac{\lambda r e^{-\lambda \bar{R}_{t}^{2}\left(\cos ^{-1}\left[\frac{r}{\bar{R}_{t}} \cos \theta\right]-\frac{r}{\bar{R}_{t}} \cos \theta \sqrt{1-\left(\frac{r}{\bar{R}_{t}} \cos \theta\right)^{2}}\right)}}{1-e^{-\lambda \pi \bar{R}_{t}^{2} / 2}} d \theta \\
& 0 \leq r \leq \bar{R}_{t}
\end{aligned}
$$

Proof: See Appendix A.

For the NFP forwarding strategy, the transmitter-receiver distance is denoted by $r_{N}$ and its distribution can be expressed by the following lemma:

Lemma 2: The distance between the transmitter and the nearest available relay device in the average transmission range $\bar{R}_{t}$ can be expressed as follows:

$$
f_{r_{N}}(r)=\frac{\lambda \pi r e^{-\lambda \pi r^{2}}}{1-e^{-\lambda \pi \bar{R}_{t}^{2} / 2}}, \quad 0 \leq r \leq \bar{R}_{t} .
$$

Proof: The distribution of distance to the nearest neighbour in Poisson networks is provided in [22]. However, note that the $p d f$ of the nearest neighbour distance has been conditioned on the probability that there is at least one available node in the forward neighbourhood of the transmitter.

Finally, for the RFP forwarding strategy, the transmitterreceiver distance is denoted by $r_{R}$ and its distribution can be expressed by the following lemma:

Lemma 3: The distance between the transmitter and a randomly selected relay inside the average transmission range of $\bar{R}_{t}$ can be expressed as follows:

$$
f_{r_{R}}(r)=\frac{2 r}{\bar{R}_{t}^{2}}, \quad 0 \leq r \leq \bar{R}_{t}
$$

\section{Proof: See Appendix B}

In the following section, we present the metric developed for performance analysis and provide the distributions of the forward packet progress.

\section{Performance Characterization}

In this section, we study the performance of the depicted system model based on the three main metrics i.e. spatial frequency reuse, transmission quality and packet forward progress. The spatial frequency reuse is quantified by the intensity of simultaneously active transmitters i.e. the number of concurrent transmissions per unit area, which is given by $\Lambda\left(\rho_{t h}\right)$. The transmission quality is measured by the probability of transmission success $\mathcal{P}_{\chi}\left(\rho_{t h}\right)$. Finally, the data dissemination is governed by the forward packet progress at each hop. The forward progress for different schemes $Z_{\chi}, \chi \in\{M, N, R\}$ is a random variable and the $p d f$ s are given by the following lemma.

Lemma 4: The per-hop forward progress achieved by using the $M F R$ forwarding strategy in a fixed average transmission range $\bar{R}_{t}$ is distributed as follows:

$$
\begin{array}{r}
f_{\mathrm{Z}_{M}}(\mathrm{z})=\frac{2 \lambda \sqrt{\bar{R}_{t}^{2}-\mathrm{z}^{2}}}{1-e^{-\lambda \pi \bar{R}_{t}^{2} / 2}} e^{-\lambda \bar{R}_{t}^{2}\left(\cos ^{-1}\left(\frac{\mathrm{z}}{\bar{R}_{t}}\right)-\left(\frac{\mathrm{z}}{\bar{R}_{t}}\right) \sqrt{1-\left(\mathrm{z} / \bar{R}_{t}\right)^{2}}\right)}, \\
0 \leq \mathrm{z} \leq \bar{R}_{t} . \quad(10)
\end{array}
$$

The per-hop forward progress achieved by using the NFP forwarding strategy in a fixed average transmission range $\bar{R}_{t}$ is distributed as follows:

$$
f_{\mathrm{Z}_{N}}(\mathrm{z})=\frac{\sqrt{2 \lambda} e^{-\lambda \pi \mathrm{z}^{2} / 2} \operatorname{erf}\left(\sqrt{\frac{\pi \lambda}{2}\left(\overline{\mathrm{R}}_{\mathrm{t}}^{2}-\mathrm{z}^{2}\right)}\right)}{1-e^{-\lambda \pi \bar{R}_{t}^{2} / 2}}, 0 \leq \mathrm{z} \leq \bar{R}_{t} .
$$

The per-hop forward progress achieved by using the RFP forwarding strategy in a fixed average transmission range $\bar{R}_{t}$ is distributed as follows:

$$
f_{\mathrm{Z}_{R}}(\mathrm{z})=\frac{4 \sqrt{\bar{R}_{t}^{2}-\mathrm{z}^{2}}}{\pi \bar{R}_{t}^{2}}, \quad 0 \leq \mathrm{z} \leq \bar{R}_{t} .
$$

\section{Proof: See Appendix C}

The average per hop forward progress denoted by $\bar{Z}_{\chi}=$ $\mathbb{E}\left[Z_{\chi}\right]$ for the three forwarding strategies can obtained using the $p d f \mathrm{~s}$ in (10), (11), and (12) for $\chi \in\{M, N, R\}$. Note that $\bar{Z}_{\chi} \sqrt{\lambda}$ can be conveniently used as a dimensionless measure of the forward progress and is therefore known as normalized average forward progress (NAFP) [23]. In order to capture the effect of spatial frequency reuse, transmission quality and packet dissemination, we define a unified performance metric called the average packet progress (APP) similar to the one introduced in [17] as follows:

$$
A P P_{\chi}\left(\rho_{t h}\right)=\mathcal{P}_{\chi}\left(\rho_{t h}\right) \times \Lambda\left(\rho_{t h}\right) \times \overline{\mathrm{Z}}_{\chi} \sqrt{\lambda},
$$

The physical meaning of APP is the average successful packet progress made per unit area in the network and the objective is to maximize the APP of the network. It is desirable as it maximizes each of the individual performance metrics. Note that for a fixed node density $\lambda$, the probability of transmission success depends on both $\chi$ and $\rho_{t h}$, the density of simultaneous transmitters depends only on $\rho_{t h}$ and the NAFP depends only on $\chi$. The challenge then is to jointly select the optimal values of $\chi$ and $\rho_{t h}$ that achieve the best balance between the spatial reuse, transmission quality and the packet dissemination given specific throughput requirements. This problem can be formulated as:

$$
\begin{array}{cc}
\max _{\chi, \rho_{t h}} & A P_{\chi}\left(\rho_{t h}\right) \\
\text { s.t. } & \frac{\lambda}{\Lambda\left(\rho_{t h}\right)} \times \frac{1}{\mathcal{P}_{\chi}\left(\rho_{t h}\right)} \times \frac{\delta}{\overline{\mathrm{Z}}_{\chi}} \times \tau \leq \epsilon
\end{array}
$$

In this case, the throughput requirement comes from the maximum allowable message transfer delay that is determined by the mission critical nature of the application in the massive IoT network. The physical meaning of the constraint is that the total transmission delay from the source to the destination should not exceed a certain threshold $\epsilon$. The term $\frac{\lambda}{\Lambda\left(\rho_{t h}\right)}$ 


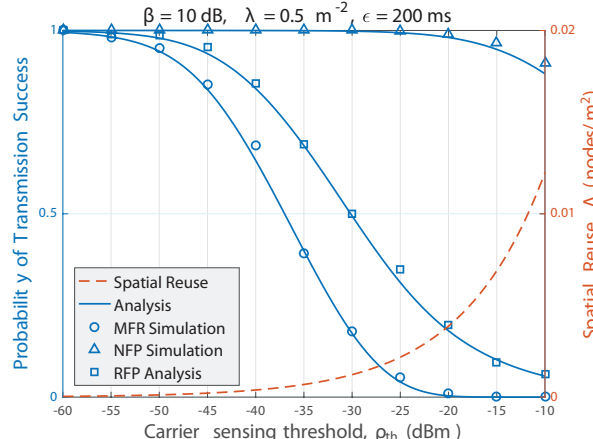

(a) Probability of transmission success and spatial reuse against carrier sensing threshold.

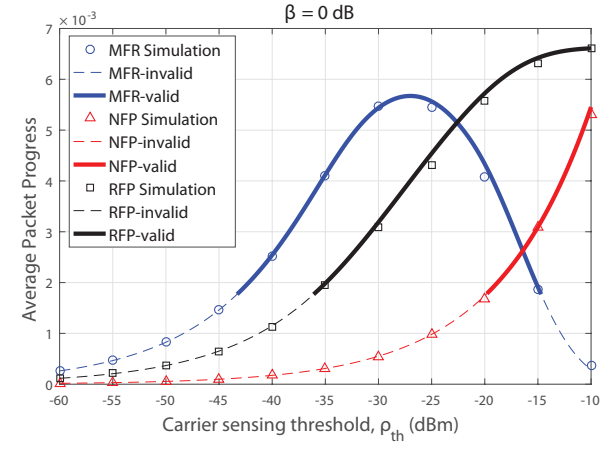

(b) APP under low QoS requirements.

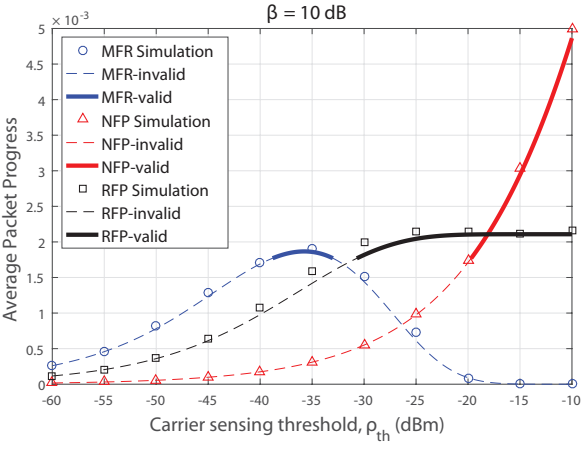

(c) APP under high QoS requirements.

Fig. 2: (a) The probability of transmission success is a decreasing function of the carrier sensing threshold as opposed to the spatial reuse, which is an increasing function. RFP can achieve the highest APP under low transmission quality requirements while NFP achieves the best APP performance under high transmission quality requirements with appropriate choice of $\rho_{t h}$.

represents the average number of time slots required by a device to win access to the channel from its neighbors, $\frac{1}{P_{s}}$ signifies the average number of re-transmissions required to achieve successful per-hop packet transmission between the transmitter and receiver, $\frac{\delta}{Z_{x}}$ represents the average number of hops that are required for the data to reach a destination device located at an arbitrary distance of $\delta$, and $\tau$ is the duration of each time slot. The goal is to find the best packet forwarding strategy and the optimal CST that maximizes the APP resulting in efficient data dissemination in mission critical massive IoT networks.

\section{Numerical Results}

In this section, we will first use a numerical example to illustrate the different tradeoffs discussed in the paper. The choice of parameters is arbitrary and is made for illustrative purposes as follows: The transmit power $P=0.1 \mathrm{~W}$, device density $\lambda=0.5$ devices $/ \mathrm{m}^{2}$, transmission range $R_{t}=10 \mathrm{~m}$ (equivalently $\rho_{\min } \approx-11 \mathrm{dBm}$ ), path-loss exponent $\eta=4$, SINR threshold $\beta=10 \mathrm{~dB}$ unless otherwise specified, distance to destination $\delta=100 \mathrm{~m}$, maximum delay tolerance $\epsilon=200$ ms and duration of time-slot $\tau=10 \mu \mathrm{s}$. Fig. 2a shows the probability of transmission success $\mathcal{P}_{\chi}$ for the three packet forwarding schemes verified through Monte Carlo simulations. It is observed that increasing the CST reduces the probability of transmission success due to the reduced interference protection around the receiver and the increased number of concurrent transmissions. As expected, the NFP scheme achieves the highest probability of success followed by $R F P$ and $M F R$ because of the increasing transmitter-receiver separation as shown in Fig 3 Fig. 2a also shows that the spatial reuse is an increasing function of the CST because increasing $\rho_{t h}$ reduces the carrier sensing range and enables more nodes to qualify for transmission though the CSMA contention.

Fig. $2 \mathrm{~b} \& 2 \mathrm{c}$ show the performance of the network under the proposed APP metric for two different QoS requirements, i.e., $\beta=0 \mathrm{~dB}$ and $\beta=10 \mathrm{~dB}$. We restrict the choice of
CST to $-11 \mathrm{~dB}$ so that the average sensing range remains greater than the average transmission range to guarantee that an interferer is not located arbitrarily close to the receiver for any of the forwarding strategies. Note that extremely high and extremely low CST results in high transmission delays. A low CST results in a high sensing range and therefore more contention, which leads to longer average time to sense an idle channel. On the other hand, a high CST results in a smaller sensing range and therefore lower interference protection, which results in more average number of retransmissions for successful message delivery. The region in which the average delay requirement is not met is represented by the dotted lines in Fig. $2 \mathrm{~b} \& \mathrm{2 \textrm {c }}$ and is labeled as invalid. It can be observed that for low QoS requirements, the RFP strategy can achieve a higher APP performance, while for high QoS requirements, the NFP can lead to the highest APP with appropriate choice of CST.

In general, the choice of best packet forwarding strategy and CST depends on several factors such as the device density, QoS requirements, and the delay tolerance. However, the fact that the RFP achieves the highest APP performance indicates that there exists a more strategic forwarding strategy in the low QoS requirements case that can result in a further improvement in performance. However, if the QoS requirement is high, short hops via the NFP strategy seems to be the best strategy.

\section{CONCLUSION}

This paper presents a framework for optimizing the mission critical data dissemination in massive IoT networks. The massive number of coexisting devices presents several performance challenges due to interference and packet delay over multi-hop transmissions. The performance tradeoffs are controlled by the carrier sensing threshold of the contention based spectrum access protocol as well as the packet forwarding strategy used at each hop. We develop an integrated framework that characterizes the performance of the system based on the spatial frequency reuse, probability of successful 


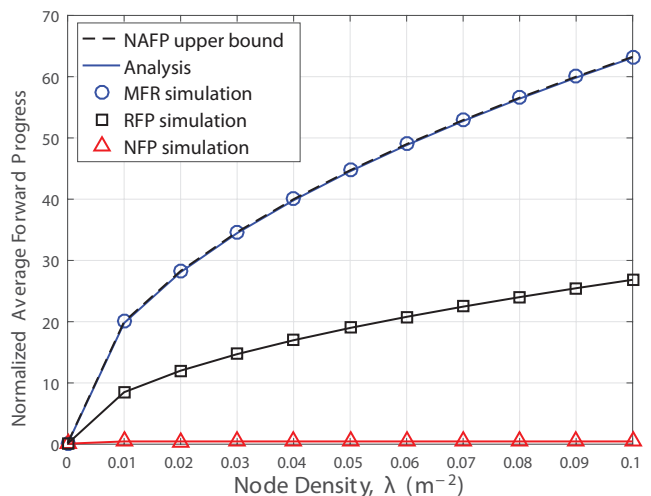

Fig. 3: The $M F R$ scheme achieves the highest per hop NAFP followed by the RFP and NFP scheme for all node densities. The difference in NAFP between the NFP and other schemes increases with the node density because of increasing number of available neighbours. The maximum possible NAFP is $R_{t} \sqrt{\lambda}$ and is therefore an upper bound.

transmission, and the forward packet progress. A combined performance metric known as the average packet progress is developed that reflects the individual performance objectives. A delay constrained optimization problem is then presented which can be used to obtain the best balance between the performance objectives. The results show that the choice of forwarding scheme and the carrier sensing threshold, that optimize the network operation, depends on the delay tolerance and QoS requirements of the network.

\section{APPENDIX A}

\section{PROOF OF LEMMA 1}

Using the approach in [23], the relay that has the most forward progress has an empty segment farther than itself. The probability of such event can be used to obtain the joint distribution between $Z_{M}$ and $D_{M}$ as shown in Fig. 11. By change of variables, i.e., $Z_{M}=r_{M} \cos \theta$ and $D_{M}=r_{M} \sin \theta$, the joint $p d f f_{r_{M}, \theta}(r, \theta)$ can be obtained. The marginal density for $r_{M}$ can then be obtained by integrating over $\theta$ from $-\frac{\pi}{2}$ to $\frac{\pi}{2}$ to obtain the result presented in Lemma 1 . Note that the $p d f$ has been conditioned on the event that there is at least one device in the forward neighbourhood of the transmitter.

\section{APPENDIX B \\ PROOF OF LEMMA 3}

The proof can be done using the cumulative distribution function $(c d f)$ approach. The $c d f$ of the random relay distance can be expressed as $F_{r_{R}}(r)=\mathbb{P}\left[r_{R} \leq r\right]=\pi r^{2} / \pi \bar{R}_{t}^{2}$. Differentiating the $c d f$ with respect to $r$ gives $f_{r_{R}}(r)$ as shown in Lemma 3

\section{APPENDix C \\ PROOF OF LEMMA 4}

The $p d f f_{\mathrm{Z}_{M}}(\mathrm{z})$ is obtained by integrating the joint density $f_{Z_{M}, D_{M}}(z, d)$, obtained in Appendix A over the range of $D_{M}$. For deriving $f_{\mathrm{Z}_{N}}(\mathrm{z})$, we can use (8) and the fact that the angle $\theta$ is independent and uniformly distributed between $-\pi / 2$ and $\pi / 2$ to obtain $f_{r_{N}, \theta}(r, \theta)$. Through transformation of variables from polar to Cartesian coordinates, we can find $f_{\mathrm{Z}_{N}, \mathrm{D}_{N}}(z, d)$. Integrating over the range of $\mathrm{D}_{N}$ proves the desired $p d f$ of $Z_{N}$. The $p d f$ of $Z_{R}$, i.e., $f_{Z_{R}}(\mathrm{z})$ can be derived using a similar procedure as the $p d f$ of $Z_{N}$.

\section{REFERENCES}

[1] A. Al-Fuqaha, M. Guizani, M. Mohammadi, M. Aledhari, and M. Ayyash, "Internet of things: A survey on enabling technologies, protocols, and applications," IEEE Commun. Surveys Tuts., vol. 17, Fourth Quarter 2015

[2] D. Evans, "The Internet of Things: How the Next Evolution of the Internet Is Changing Everything." Cisco Inc., White Paper, 2011.

[3] The Internet Of Things: Mapping the Value Beyond the Hype. Mckinsey Global Institute, 2015.

[4] "Massive IoT in the City." Ericsson Mobility Report, Ericsson, Nov. 2016.

[5] "Internet of Things forecast." Ericsson Mobility Report, Ericsson, Nov. 2016.

[6] O. Bello and S. Zeadally, "Intelligent device-to-device communication in the internet of things," IEEE Systems J., vol. 10, Sep. 2016.

[7] V. Gazis, "A survey of standards for machine to machine (M2M) and the internet of things (IoT)," IEEE Commun. Surveys Tuts., 2016.

[8] M. J. Farooq and Q. Zhu, "Secure and reconfigurable network design for critical information dissemination in the internet of battlefield things (IoBT)," in 15th Intl Symp. Model. Optim. Mobile, Ad Hoc, Wireless Netw., (WiOpt 2017), Paris , France 2017.

[9] C. Tang, L. Song, J. Balasubramani, S. Wu, S. Biaz, Q. Yang, and $\mathrm{H}$. Wang, "Comparative investigation on CSMA/CA-based opportunistic random access for internet of things," IEEE Internet Things J., vol. 1, April 2014.

[10] J. Zhu, X. Guo, L. L. Yang, W. S. Conner, S. Roy, and M. M. Hazra, "Adapting physical carrier sensing to maximize spatial reuse in 802.11 mesh networks: Research Articles," Wirel. Commun. Mob. Comput., vol. 4, pp. 933-946, Dec. 2004.

[11] Y. Kim, F. Baccelli, and G. de Veciana, "Spatial reuse and fairness of ad hoc networks with channel-aware CSMA protocols," IEEE Trans. Inf. Theory, vol. 60, Jul. 2014.

[12] F. Baccelli and B. Bllaszczyszyn, "Stochastic Geometry and Wireless Networks: Volume II Applications," Found. Trends Netw., vol. 3, pp. 249-449, Mar. 2009.

[13] P. Suriyachai, U. Roedig, and A. Scott, "A survey of MAC protocols for mission-critical applications in wireless sensor networks," IEEE Commun. Surveys Tuts., vol. 14, Second Quarter 2012.

[14] G. Alfano, M. Garetto, and E. Leonardi, "New directions into the stochastic geometry analysis of dense CSMA networks," IEEE Trans. Mobile Comput., vol. 13, Feb. 2014.

[15] H. ElSawy and E. Hossain, "A modified hard core point process for analysis of random CSMA wireless networks in general fading environments," IEEE Trans. Commun., vol. 61, Apr. 2013.

[16] H. Q. Nguyen, F. Baccelli, and D. Kofman, "A stochastic geometry analysis of dense IEEE 802.11 networks," in 26th IEEE Intl. Conf. Comput. Commun. (INFOCOM 2007), Alaska, USA, May 2007.

[17] M. J. Farooq, H. ElSawy, and M. S. Alouini, "A stochastic geometry model for multi-hop highway vehicular communication," IEEE Trans Wireless Commun., vol. 15, Mar. 2016.

[18] A. T. Giang, A. Busson, and M. D. Renzo, "Modeling and optimization of CSMA/CA in vanet," Annals of Operations Research, vol. 239, no. 2, 2016.

[19] P. Nardelli, P. Cardieri, and M. Latva-aho, "Efficiency of wireless networks under different hopping strategies," IEEE Trans. Wireless Commun., vol. 11, Jan. 2012

[20] M. J. Farooq, H. ElSawy, and M. S. Alouini, "Modeling inter-vehicle communication in multi-lane highways: A stochastic geometry approach," in IEEE 82nd Vehicular Technology Conference (VTC-Fall 2015), pp. 1-5, Boston, USA, Sep. 2015.

[21] M. Haenggi, "Mean interference in hard-core wireless networks," IEEE Commun. Lett., vol. 15, Aug. 2011.

[22] M. Haenggi, "On distances in uniformly random networks," IEEE Trans. Inf. Theory, vol. 51, Oct. 2005.

[23] T.-C. Hou and V. Li, "Transmission range control in multihop packet radio networks," IEEE Trans. Commun., vol. 34, Jan. 1986. 